\title{
Framed ALOHA Protocol with FIFO-Blocking and LIFO-Push out Discipline
}

\author{
Vicente Casares Giner, Víctor Sempere Payá, Davíd Todolí Ferrandis \\ Departamento de Comunicaciones, Universidad Politécnica de Valencia \\ Camino Vera s/n, 46022, Valencia (Spain) \\ Corresponding author: E-mail: vcasares@dcom.upv.es
}

Received: April 29, 2014

Accepted: June 15, 2014

Published: August 14, 2014

DOI: 10.5296/npa.v6i3.5557

URL: http://dx.doi.org/10.5296/ npa.v6i3.5557

\begin{abstract}
This paper considers the framed ALOHA protocol for a finite number of terminals. Terminals are single-buffered, i.e. each one can store only one data packet. We derive the throughput and the delay under two types of queue disciplines, first-in-first-out (FIFO) and last-in-first-out (LIFO). It is assumed that terminals are independent each other and generate packets of constant size according to a Bernoulli process. The one slot duration equals to the one packet transmission. The analysis is carried out by means of Markovian tools. Some discussion on the applicability of that protocol is addressed.
\end{abstract}

Keywords: Framed ALOHA, Queue Discipline, FIFO, LIFO, Push out, Drop-out Probability. 


\section{Introduction}

When two or more terminals have to share a single wireless channel, quite often a random access protocol is pointed out as a common solution. The seminal work by Abramson [1] is considered as the birth of this type of protocols. Two years later, the slotted version of ALOHA protocol was published [2] with the main attribute to double the throughput of the original ALOHA. Since then, many ALOHA type protocols have been formulated and published in the open literature [3] with the main two goals of gaining more throughput and achieve better stability. ALOHA and its variants appears to be quite suitable to accommodate burst traffic. Even though the throughput of the ALOHA family protocols is rather low, due to its low complexity and no other solution for some wireless scenarios, ALOHA protocols are used in many wireless systems, such as in the GSM cellular $(2 \mathrm{G})$ and further generations, by which the mobile terminal (MT) access for the first time to the base station [4].

In this paper we have dealt with the framed S-ALOHA protocol [5]. The frame structure is depicted in Fig. 1, where the frame is composed by $V$ slots. The duration of one time slot equals to the transmission time of one data packet. According to [6], several reasons invite to its implementation. For instance, it can be more efficient to send the ACKs one per frame instead of one per time slot. Also, framed S-ALOHA offers the possibility to distribute the set of $V$ slots per frame in a grouping basis, in proportion to the traffic generated per group.

In the present work we overview the framed S-ALOHA for a finite number of terminals, where each one has a buffer size equal to one. Then, two main results are reported. First, we provide some new recurrences for calculus of the conditional probabilities, $E(m, k, V)$,

$S(m, k, V)$ and $C(m, k, V)$, Assuming we perform a random allocation of $m$ data packets in

$V$ slots, $k$ of them results empty, $E(m, k, V)$, with a single data packet, $S(m, k, V)$, or with

more than a single data packet, $C(m, k, V)$. Second, under the two queue disciplines first-in-first-out (FIFO) and last-in-first-out (LIFO), we derive the Cumulative Distribution Functions $(\mathrm{CDF})$ of the sojourn time for data packet that are successfully transmitted or are dropped out.

The structure of the paper is as follows. After this short introduction, section 2 deals with the Markov model of the scenario under consideration. Section 3 concentrates on the CDFs. Results are presented and discussed in section 4. Finally conclusions are reported in section 5.

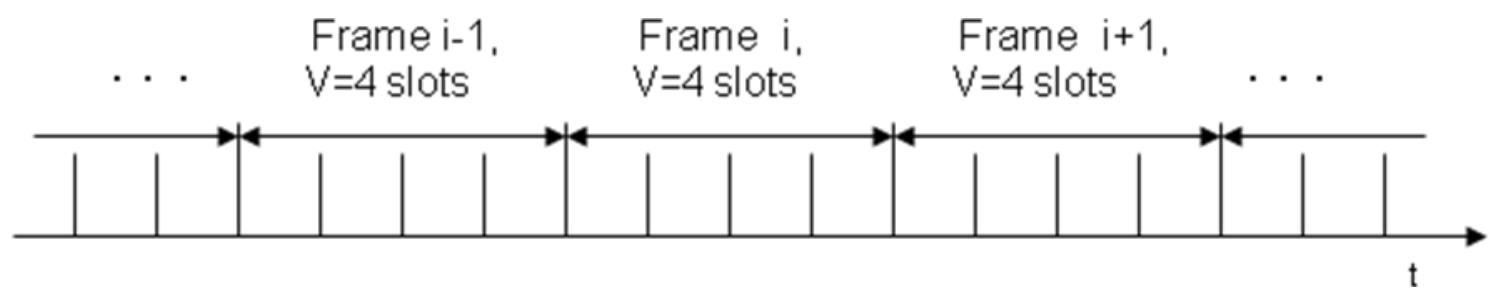

Figure 1. Frame structure with $V=4$ slots per frame. 


\section{System modeling}

We consider a finite number of $M$ terminals that are sharing a single wireless channel with a Time Division Multiple Access (TDMA) structure. As Fig. 1 shows, time is slotted, with $V$ slots per frame. Each terminal generates data packets according to a Bernoulli model. In other words, for a given terminal and a given time slot, with probability $p_{a c t}$, a single data packet is generated and with probability $1-p_{a c t}$, no data packets are generated. Each terminal has its own data buffer of size equal to 1 . Then, upon the generation or arrival of one data packet, the packet occupies the single position in the buffer if it is find empty. Otherwise we consider two disciplines, FIFO-BL (FIFO-Blocking) and LIFO-PO (LIFO-Push out) [7]. In the first discipline, the packet is dropped-out and in the second case, the new data packet pushes out the oldest one in the buffer and joins the line.

Following Fig. 1, a frame of $V$ consecutive slots has been defined. Then, for a given terminal, the probability that at least one data packet is generated during a frame is given by $a=1-\left(1-p_{a c t}\right)^{V}$. Then, the random access protocol acts as follows. Let us consider that, at the beginning of a given frame, we have $i$ data packets ready for transmission, that is, $i$ buffers each one with one data packet. Then, with probability $r$ each packet will choose the actual frame to try the access and with probability $1-r$ the packet will defer the access to the next frame. The permission (or access) probability $r$ could be estimated by means of some centralized control and broadcasted to all terminals in the system [8]. Then, the probability that $j$ data packets $(0 \leq j \leq i)$ get permission to access is given by a binomial distribution, $B(i, j, r)$, Eq. (A.2). Then, each of the $j$ terminals that gain the permission to access will choose one of the $V$ slots with probability $1 / V$. Let $S(j, k, V)$ denote the probability that $k$ among $j$ data packets gain a successful access, $0 \leq k \leq j$. Joining both actions in a single expression we have

$$
D_{k}^{i}(V, r)=\sum_{j=k}^{i} B(i, j, r) S(j, k, V)
$$

Where $D_{k}^{i}(V, r)$ is the probability to have $k$ successful transmissions from a total of $i$ data packets ready to transmit at the beginning a given frame. In the sequel, in some occasions, we will use $D_{k}^{i}$ as a short notation of $D_{k}^{i}(V, r)$.

Clearly, a Markov chain can be defined at the beginning of each frame. We denote by $\pi_{i}$ 


\section{Macrothink}

Network Protocols and Algorithms

ISSN 1943-3581

2014, Vol. 6, No. 3

the associated probability in steady state of that Markov chain which indicates the probability to have $i$ data packets ready for transmission at the time instants of observation. And let $P_{i, j}$ denote the conditional probabilities that define that Markov chain. $P_{i, j}$ is the conditional probability that, assuming we have $i$ data packets ready for transmission at the beginning of a given frame, at the beginning of the next frame we will have $j$ data packets ready for transmission. Then $P_{i, j}$ is given by

$$
P_{i, j}=\sum_{k=\min (0, i-j)}^{i} D_{k}^{i}(V, r) A(M-i+k, j-i+k, a)
$$

Where $A(m, n, a)$ follows a binomial distribution and takes into account the arrival process. $A(m, n, a)$ is the probability that in a given frame of $V$ slots, $n$ out of $m$ terminals each one generates a data packet. Notice that, for a given terminal, the probability to generate at least one data packet per frame, $a=1-\left(1-p_{a c t}\right)^{V}$, increases with $V$ for a fixed $p_{a c t}$. If a given terminal generates more than one data packet per frame, only one of them is considered, and the other are not considered. Clearly, the steady state probabilities $\pi=\left[\pi_{0}, \pi_{1}, \pi_{2}, \ldots . . \pi_{M}\right]$ are obtained by solving the system

$$
\boldsymbol{\pi}=\boldsymbol{\pi} \mathbf{P}
$$

, with the normalization condition of $\boldsymbol{\pi} \mathbf{1}=1$, where $\mathbf{1}$ is a column vector with all its elements equal to 1 .

\section{System observed by a tagged data packet}

In this section we deal with a data packet, our tagged packet that is generated by a given terminal, our tagged terminal, and it is offered to the system. We derive the dropping probability and the sojourn time of our tagged packet in the system under the already described FIFO-BL and LIFO-PO disciplines. To that purpose we will use the BASTA property (Bernoulli Arrivals See Time Averages) for slotted systems, as the dual property of PASTA (Poisson Arrivals See Time Averages) for continuous systems. Then, according to BASTA property, when our tagged packet arrives to the system, with probability $\pi_{k}$ it will find $k$ data packets. Upon the packet arrival, we can distinguish three different possible cases:

\subsection{Case A}


With probability $(M-k) / M$ our tagged data packet will find its buffer empty, that is, it will be admitted with probability

$$
P_{A}(M, k)=\frac{M-k}{M}
$$

In case of FIFO_BL discipline, our tagged data packet will remain in its buffer until it is successfully transmitted. In case of LIFO-PO discipline, our tagged data packet has the risk to, before being served, be pushed out by another data packet coming from our tagged terminal.

\subsection{Case B}

With probability $k / M$ our tagged packet will find its buffer occupied by a packet that previously was originated by our tagged terminal. But this predecessor packet can be successfully transmitted with probability $l / k$, where $l$ is the number of packets out of $k$ packets that, with probability $D_{l}^{k}(V, r)$ are transmitted in the actual frame. It means that the predecessor packet will exit the system and will empty the one size buffer, remaining available for our tagged packet. The probability of this event is

$$
P_{B}(M, k, l)=\frac{k}{M} \frac{l}{k} D_{l}^{k}(V, r)=\frac{l}{M} D_{l}^{k}(V, r)
$$

Similar to Case A, for FIFO_BL discipline, our packet will remain in its buffer until it is successfully transmitted. For LIFO-PO discipline, our tagged packet has the risk to, before being served, be pushed out by another packet coming from our tagged terminal.

\subsection{Case C}

This case complements the Case B. With probability $k / M$ our tagged packet will find its buffer occupied by a packet previously originated by our tagged terminal. But this predecessor packet can fail in its transmission attempt, and this can happen with probability $k-l / k$. Therefore, the probability of this event is

$$
P_{C}(M, k, l)=\frac{k}{M} \frac{k-l}{k} D_{l}^{k}(V, r)=\frac{k-l}{M} D_{l}^{k}(V, r)
$$

Then, our tagged packet will be dropped out when FIFO-BL discipline is implemented, or will push out the packet in the buffer when LIFO-PO is implemented.

We remark the fact that in FIFO-BL discipline, once a given packet is accepted in its buffer, soon or later it will be successfully transmitted. Then, from the previous arguments, for FIFO-BL discipline we can derive the probability that our tagged packet be admitted and later on be successfully transmitted. This probability is given by 


$$
P_{A F}=\sum_{k=0}^{M}\left[P_{A}(M, k)+\sum_{l=0}^{k} P_{B}(M, k, l)\right] \pi_{k}
$$

On the other hand, the probability to reject our tagged packet using the FIFO-BL discipline is given by

$$
P_{R F}=\sum_{k=0}^{M} \sum_{l=0}^{k} P_{C}(M, k, l) \pi_{k}
$$

Obviously, from (4), (5) and (6), or alternatively from (7) and (8) it can be seen that $P_{A F}+P_{R F}=1$.

We just mention the fact that from the above results we can derive some mean or average values. For instance, having in mind the FIFO-BL discipline, let $M n_{R F}$ denote the mean number of data packet that, originated in our tagged terminal, are rejected during the waiting time in the buffer of our tagged data packet. Clearly $P_{A F}$ and $M n_{R F}$ satisfy the relationship:

$$
P_{A F}=\frac{1}{M n_{R F}+1} \quad \rightarrow \quad M n_{R F}=\frac{1}{P_{A F}}-1
$$

From (9), the mean waiting time of our tagged packet, $M w_{F}$, can be obtained from the following arguments. First, because of the memory-less property of the Bernoulli arrival process, the number of time slots between two consecutive data packets generated by a given terminal follows a geometric law with, with mean value equal to $1 / a$, with $a=1-\left(1-p_{a c t}\right)^{V}$. Second, we keep in mind the fact that, if another packet from our tagged terminal is generated in the same frame that our tagged packet is being successfully transmitted; such a new packet will be admitted in the buffer. Therefore we can express $M w_{F}$ as

$$
M w_{F}=\frac{M n_{R F}}{a}+1
$$

With regard to LIFO-PO discipline, at first glance any packet is initially admitted into the buffer of size 1 . Then, a given packet is pushed out by some forthcoming packet from our tagged terminal or it is successfully transmitted, whichever occurs first. Let $P_{R L}$, respectively 
$P_{A L}$, denote the probability that our tagged packet being pushed out or finally be successfully transmitted. At this point we claim to the fact that, regardless of the FIFO-BL or LIFO-PO queue disciplines, the throughput of the system is the same. Therefore we can conclude that $M n_{R L}=M n_{R F}$ and that $M n_{A L}=M n_{A F}$, where $M n_{R L}$, respectively $M n_{A L}$, denote the mean number of data packets that, originated in our tagged terminal, are rejected, respectively successfully transmitted under LIFO-PO discipline. Other mean values are derived in the next lines. Furthermore, for both FIFO-BL and LIFO-PO queue disciplines, we obtain closed expressions for the cumulative distribution functions (CDFs) of the corresponding sojourn times.

\subsection{Sojourn time distribution for FIFO-BL and LIFO-PO disciplines}

Here we derive the sojourn time distributions for the mentioned queue disciplines. In fact, those distributions are Phase-type distributions, in the sequel PH, represented as $(\boldsymbol{\alpha}, \mathbf{T})[9]$, [10] where $\alpha$ is the initial vector of probabilities and $\mathbf{T}$ reflects the transition probabilities between transient states. Also, $\mathbf{T}^{0}=\mathbf{1}-\mathbf{T} \mathbf{1}$ is the column vector that contains the transition probabilities from transient states to the absorbing state. For FIFO-BL, respectively LIFO-PO discipline we identify, $\left(\boldsymbol{\alpha}_{F}, \mathbf{T}_{F}\right), \mathbf{T}_{F}^{0}$, respectively $\left(\boldsymbol{\alpha}_{L}, \mathbf{T}_{L}\right), \mathbf{T}_{L}^{0}$. In the next lines we derive the vectors and matrices of both PHs.

\subsubsection{Initial states for FIFO-BL discipline.}

For FIFO-BL discipline, our tagged packet is admitted into the system in cases A and B, respectively, subsection 3.1 and 3.2 and rejected in case $C$. When in cases A and B, our tagged packet will compete for the medium access together with other packets. Probabilistically, the number of those packets can be estimated according to $a$ ) the number of packets pending to be transmitted and $b$ ) the number of packets that together with our tagged packet have arrived to the system. Then, considering cases A and B, we have

Case A: From Eq. (4) the probability that our tagged packet compete for the first time with other $j$ packets $\left(j=0,1, \ldots M^{\prime}=M-1\right)$ is given by

$$
P_{A-F}(M, k, j)=P_{A}(M, k) \sum_{l=\max (0, k-j)}^{k} D_{l}^{k}(V, r) A\left(M^{\prime}-k+l, j-k+l, a\right)
$$

Case B: From Eq. (5) the probability that our tagged data packet compete for the first time with other $j$ packets $\left(j=0,1, \ldots M^{\prime}=M-1\right)$ is given by 


$$
P_{B-F}(M, k, j)=\sum_{l=\max (0, k-j)}^{k} P_{B}(M, k, l) A\left(M^{\prime}-k+l, j-k+l, a\right)
$$

From (11) and (12) and using the BASTA property, we obtain the probability that our tagged packet will compete for the first time in the wireless access together with other $j$ packets $\left(j=0,1, \ldots M^{\prime}=M-1\right)$. It is given by

$$
\boldsymbol{\alpha}_{F ; j}=\sum_{k=0}^{M}\left(P_{A-F}(M, k, j)+P_{A-F}(M, k, j)\right) \pi_{k}
$$

In vector notation we write (13)

$$
\boldsymbol{\alpha}_{F}=\left[\boldsymbol{\alpha}_{F ; 0}, \boldsymbol{\alpha}_{F ; 1}, \ldots, \boldsymbol{\alpha}_{F ; M^{\prime}}\right] \text { with } M^{\prime}=M-1
$$

Then, $\boldsymbol{\alpha}_{F ; M}=1-\boldsymbol{\alpha}_{F} \mathbf{1}$ is the blocking probability and is coincident with the probability that at time $t=0^{+}$the system be in the absorbing state. States $0,1, \cdots M^{\prime}$ are identified as transient states and state $M=M^{\prime}+1$ as absorbing state. That is,

$$
\boldsymbol{\alpha}_{F} \mathbf{1}=1-\boldsymbol{\alpha}_{F ; M}=P_{A F}=1-P_{R F}
$$

\subsubsection{Initial states for LIFO-PO discipline.}

Clearly, for LIFO-PO discipline, all packets offered to the system are initially admitted. From the description of cases $\mathrm{A}$ and $\mathrm{B}$, our tagged packet did not push out any previous data packet. In case $\mathrm{C}$, our tagged packet did push out the already previous packet found in the buffer upon its arrival. Then, considering all three cases we have

Cases A and B: Regardless of the queue discipline, every packet is admitted, so LIFO-PO and FIFO-BL disciplines have the same treatment.

Case C: From Eq. (6) the probability that our tagged packet will compete for the first time with other $j$ packets $\left(j=0,1, \ldots, M^{\prime}=M-1\right)$ is given by

$$
P_{C-L}(M, k, j)=\sum_{l=\max (0, k-j)}^{k} P_{C}(M, k, l) A\left(M^{\prime}-k+l, j-k+l, a\right)
$$

According to BASTA property we weight (16) with the steady state probabilities, and 
the result becomes

$$
\boldsymbol{\alpha}_{C ; j}=\sum_{k=0}^{M} P_{C-L}(M, k, j) \pi_{k}
$$

Then, joining all three cases we have for LIFO-PO that

$$
\alpha_{L ; j}=\alpha_{F ; j}+\alpha_{C ; j}
$$

And in vector notation

$$
\begin{aligned}
& \boldsymbol{\alpha}_{C}=\left[\boldsymbol{\alpha}_{C ; 0}, \boldsymbol{\alpha}_{C ; 1}, \ldots, \boldsymbol{\alpha}_{C ; M^{\prime}}\right] \\
& \boldsymbol{\alpha}_{L}=\boldsymbol{\alpha}_{F}+\boldsymbol{\alpha}_{C}
\end{aligned}
$$

Here we remark the fact that $\boldsymbol{\alpha}_{F}$, Ec. (14) is a sub-stochastic vector, $\boldsymbol{\alpha}_{F} \mathbf{1}<1$, and $\boldsymbol{\alpha}_{L}$, Ec. (20) is a stochastic vector, $\boldsymbol{\alpha}_{L} \mathbf{1}=\boldsymbol{\alpha}_{F} \mathbf{1}+\boldsymbol{\alpha}_{C} \mathbf{1}=1$.

Obviously, also we can write

$$
\boldsymbol{\alpha}_{C} \mathbf{1}=1-\boldsymbol{\alpha}_{F} \mathbf{1}=\boldsymbol{\alpha}_{F ; M}=P_{R F}=1-P_{A F}
$$

\subsubsection{Matrices $\mathbf{T}$ and $\mathbf{T}^{0}$ for FIFO-BL.}

With regards to the components of $\mathbf{T}_{F}$ - the sub-index stands from FIFO- let $T_{L ; i, j}$ be the conditional probability that, assuming that our tagged packet is going to compete for the access with other $i$ packets, it will collision with other(s) packet(s) and in the next frame it will try to gain the access in competition with other $j$ packets. We can write

$$
T_{F ; i, j}=\sum_{k=\max (0, i-j)}^{i}\left(1-\frac{k}{i+1}\right) D_{k}^{i+1}(V, r) A_{k-i+j}^{M-i-1+k}
$$

In (22), $D_{n}^{m}(V, r)$ has been defined in (1) and $A_{n}^{m}=B(m, n, a)$ is the binomial distribution; e.g., the probability that $n$ out of $m$ terminals each one generates a single data packet. We recall the fact that each terminal can generate up to $V$ data packets in one frame, one per slot, but only one is offered to the system and the others are not considered.

With regards to $\mathbf{T}_{F}^{0}$, let $T_{F ; i}^{0}$ be the conditional probability that our tagged packet will not collide with other packet(s), so it is successfully transmitted. This probability is given by 


$$
T_{F ; i}^{0}=\sum_{k=1}^{i+1} \frac{k}{i+1} D_{k}^{i+1}(V, r)
$$

In matrix notation, (22) and (23) can be written as, respectively

$$
\mathbf{T}_{F}=\left[\begin{array}{ccccc}
D_{1}^{1} A_{0}^{M^{\prime}} & D_{1}^{1} A_{1}^{M^{\prime}} & D_{1}^{1} A_{2}^{M^{\prime}} & \cdots & D_{1}^{1} A_{M^{\prime}}^{M^{\prime}} \\
\frac{D_{1}^{2} A_{0}^{M^{\prime}}}{2} & \sum_{k=0}^{1} \frac{2-k}{2} D_{k}^{2} A_{k}^{M^{\prime}-1+k} & \sum_{k=0}^{1} \frac{2-k}{2} D_{k}^{2} A_{k+1}^{M^{\prime}-1+k} & \cdots & \sum_{k=0}^{1} \frac{2-k}{2} D_{k}^{2} A_{k+M^{\prime}-1}^{M^{\prime}-1+k} \\
\frac{D_{2}^{3} A_{0}^{M^{\prime}}}{3} & \sum_{k=1}^{2} \frac{3-k}{3} D_{k}^{3} A_{k-1}^{M^{\prime}-2+k} & \sum_{k=0}^{2} \frac{3-k}{3} D_{k}^{3} A_{k}^{M^{\prime}-2+k} & \cdots & \sum_{k=0}^{2} \frac{3-k}{3} D_{k}^{3} A_{k+M^{\prime}-2}^{M^{\prime}-2+k} \\
\frac{D_{3}^{4} A_{0}^{M^{\prime}}}{4} & \sum_{k=2}^{3} \frac{4-k}{4} D_{k}^{4} A_{k-2}^{M^{\prime}-3+k} & \sum_{k=1}^{3} \frac{4-k}{4} D_{k}^{4} A_{k-1}^{M^{\prime}-3+k} & \cdots & \sum_{k=0}^{3} \frac{4-k}{4} D_{k}^{4} A_{k+M^{\prime}-3}^{M^{\prime}-2+k} \\
\vdots & \vdots & \vdots & \ddots & \vdots
\end{array}\right]
$$

$$
\mathbf{T}_{F}^{0}=\left[D_{1}^{1}, \sum_{k=1}^{2} \frac{k}{2} D_{k}^{2}, \cdots, \sum_{k=1}^{M} \frac{k}{M} D_{k}^{M}\right]
$$

, where in (24) and (25) it is used the short notation $D_{n}^{m}=D_{n}^{m}(V, r)$. Then, expressing the sojourn time of our tagged packet in terms of number of frames, the corresponding generating function, $R_{F}(z)$, becomes

$$
R_{F}(z)=\boldsymbol{\alpha}_{M}+z \boldsymbol{\alpha}_{F}\left[\mathbf{I}-z \mathbf{T}_{F}\right]^{-1} \mathbf{T}_{F}^{0}
$$

\subsubsection{Matrices $\mathbf{T}$ and $\mathbf{T}^{0}$ for LIFO-PO.}

With regards to the components of $\mathbf{T}_{L}$ - the sub-index stands from LIFO- let $T_{L ; i, j}$ be the conditional probability that, assuming that our tagged packet is going to compete for the access with other $i$ packets, it will collide with other(s) packet(s) and in the next frame it will try to gain the access in competition with other $j$ data packets, $j=0,1, \cdots, M^{\prime}=M-1$. Here we take into account that no packets from our tagged terminal will arrive during the frame of $V$ slots. Then it is straightforward to see that

$$
T_{L ; i, j}=(1-a) T_{F ; i, j}
$$

Alternatively, our tagged packet will be pushed out with probability $a \sum_{j=0}^{M^{\prime}} T_{F ; i, j}$. On the 


\section{Macrothink}

Network Protocols and Algorithms

ISSN 1943-3581

2014, Vol. 6, No. 3

other hand, the probability that our tagged packet be successfully transmitted is also given by (23). Then, with regards to $\mathbf{T}_{L}^{0}$, let $T_{L ; i}^{0}$ be the conditional probability that our tagged packet does not collide with other data packet(s), that is, it is successfully transmitted. $T_{L ; i}^{0}$ is given by

$$
T_{L ; i}^{0}=T_{F ; i}^{0}+a \sum_{j=0}^{M^{\prime}} T_{F ; i, j}
$$

In matrix notation, (27) and (28) can be written as, respectively, $\mathbf{T}_{L}=(1-a) \mathbf{T}_{F}$ and $\mathbf{T}_{L}^{0}=\mathbf{T}_{F}^{0}+a \mathbf{T}_{F} \mathbf{1}$.Then, since we know that $\alpha_{L ; M}=0$ (the probability that our system be in the absorbing state at time $t=0^{+}$is zero), we write for the corresponding generating function, $R_{L}(z)$ results to be

$$
R_{L}(z)=\alpha_{L ; M}+z \boldsymbol{\alpha}_{L}\left[\mathbf{I}-z \mathbf{T}_{L}\right]^{-1} \mathbf{T}_{L}^{0}=z \boldsymbol{\alpha}_{L}\left[\mathbf{I}-z(1-a) \mathbf{T}_{F}\right]^{-1}\left[\mathbf{T}_{F}^{0}+a \mathbf{T}_{F} \mathbf{1}\right]=R_{A L}(z)+R_{R L}(z)
$$

$\operatorname{In}(29) R_{A L}(z)=z \boldsymbol{\alpha}_{L}\left[\mathbf{I}-z(1-a) \mathbf{T}_{F}\right]^{-1} \mathbf{T}_{F}^{0}$, respectively $R_{R L}(z)=z \boldsymbol{\alpha}_{L}\left[\mathbf{I}-z(1-a) \mathbf{T}_{F}\right]^{-1} a \mathbf{T}_{F} \mathbf{1}$, is the generating function of the random variable (r.v.) number of frames that our tagged packet is in the buffer before it is successfully transmitted, respectively pushed out.

Based on the previous results, in the sequel we denote $\mathbf{T}_{F}=\mathbf{T}$ and $\mathbf{T}_{F}^{0}=\mathbf{T}^{0}$ such that

$$
\begin{aligned}
& R_{F}(z)=R_{A F}(z)+R_{R F}(z) ; \text { with } R_{A F}(z)=z \boldsymbol{\alpha}_{F}[\mathbf{I}-z \mathbf{T}]^{-1} \mathbf{T}^{0} \text { and } R_{R F}(z)=\boldsymbol{\alpha}_{M} \\
& R_{L}(z)=R_{A L}(z)+R_{R L}(z) ; \text { with } \\
& \quad R_{A L}(z)=z \boldsymbol{\alpha}_{L}[\mathbf{I}-z(1-a) \mathbf{T}]^{-1} \mathbf{T}^{0} \text { and } R_{R F}(z)=z \boldsymbol{\alpha}_{L}[\mathbf{I}-z(1-a) \mathbf{T}]^{-1} a \mathbf{T} \mathbf{1}
\end{aligned}
$$

Obviously we have $R_{F}(1)=1$ and $R_{L}(1)=1$. Notice that in connection with (7) and (8), the probabilities that our tagged packet be successfully transmitted or pushed out, are given by, respectively

$$
\begin{aligned}
& P_{A d m}=R_{A F}(1)=P_{A F}=R_{A L}(1)=P_{A L} \\
& P_{\operatorname{Re} j}=R_{R F}(1)=P_{R F}=R_{R L}(1)=P_{R F}
\end{aligned}
$$




\section{Macrothink}

Factorial moments can easily be obtained from the successive derivatives of (30) and (31) evaluated at $z=1$.

$$
R_{F}^{(k)}(1)=R_{A F}^{(k)}(1)=k ! \boldsymbol{\alpha}_{F} \mathbf{T}^{k-1}[\mathbf{I}-\mathbf{T}]^{-k} \mathbf{1}
$$

, and

$$
\begin{aligned}
& R_{A L}^{(k)}(1)=k !(1-a)^{k-1} \boldsymbol{\alpha}_{L} \mathbf{T}^{k-1}[\mathbf{I}-(1-a) \mathbf{T}]^{-(k+1)} \mathbf{T}^{0} \\
& R_{R L}^{(k)}(1)=k !(1-a)^{k-1} \boldsymbol{\alpha}_{L} \mathbf{T}^{k-1}[\mathbf{I}-(1-a) \mathbf{T}]^{-(k+1)} a \mathbf{T} \mathbf{1}
\end{aligned}
$$

The mean values are obtained from the above expressions for $k=1$ :

$$
\begin{aligned}
& R_{A F}^{(1)}(1)=\alpha_{F}[\mathbf{I}-\mathbf{T}]^{-1} \mathbf{1} \\
& R_{A L}^{(1)}(1)=\alpha_{L}[\mathbf{I}-(1-a) \mathbf{T}]^{-2} \mathbf{T}^{0} \\
& R_{R L}^{(1)}(1)=\alpha_{L}[\mathbf{I}-(1-a) \mathbf{T}]^{-2} a \mathbf{T} \mathbf{1}
\end{aligned}
$$

Remark: we observe that, from the principle of conservation of work, it is fulfilled that $R_{A F}^{(1)}(1)=R_{A L}^{(1)}(1)+R_{R L}^{(1)}(1)$ and having in mind the fact that $[\mathbf{I}-(1-a) \mathbf{T}]^{-1}\left[\mathbf{T}^{0}+a \mathbf{T} \mathbf{1}\right]=\mathbf{1}$, Eq. (40) can be written as $\boldsymbol{\alpha}_{F}[\mathbf{I}-\mathbf{T}]^{-1} \mathbf{1}=\boldsymbol{\alpha}_{L}[\mathbf{I}-(1-a) \mathbf{T}]^{-1} \mathbf{1}$.

\section{Results and applicability}

Some illustrative results are reported in this section, for a number of terminals equal to $M=8$ and a frame size of $V=5$ slots. Fig. 2 and 3 show the CDFs of the sojourn time, in number of frames, for data packets successfully transmitted under FIFO-BL and LIFO-PO disciplines. For a permission probability $r=0.75$, Fig. 2 plots the results for several values of

$$
p_{a c t}=0.01,0.05,0.1,0.15,0.20 \quad \text { equivalent }
$$

to

$a=1-\left(1-p_{a c t}\right)^{V}=0.049,0.226,0.409,0.556,0.672$. In Fig. 3 we have fixed $p_{a c t}=0.05$ and the permission probability is set to $r=0.2,0.4,0.6,0.8,1$. 


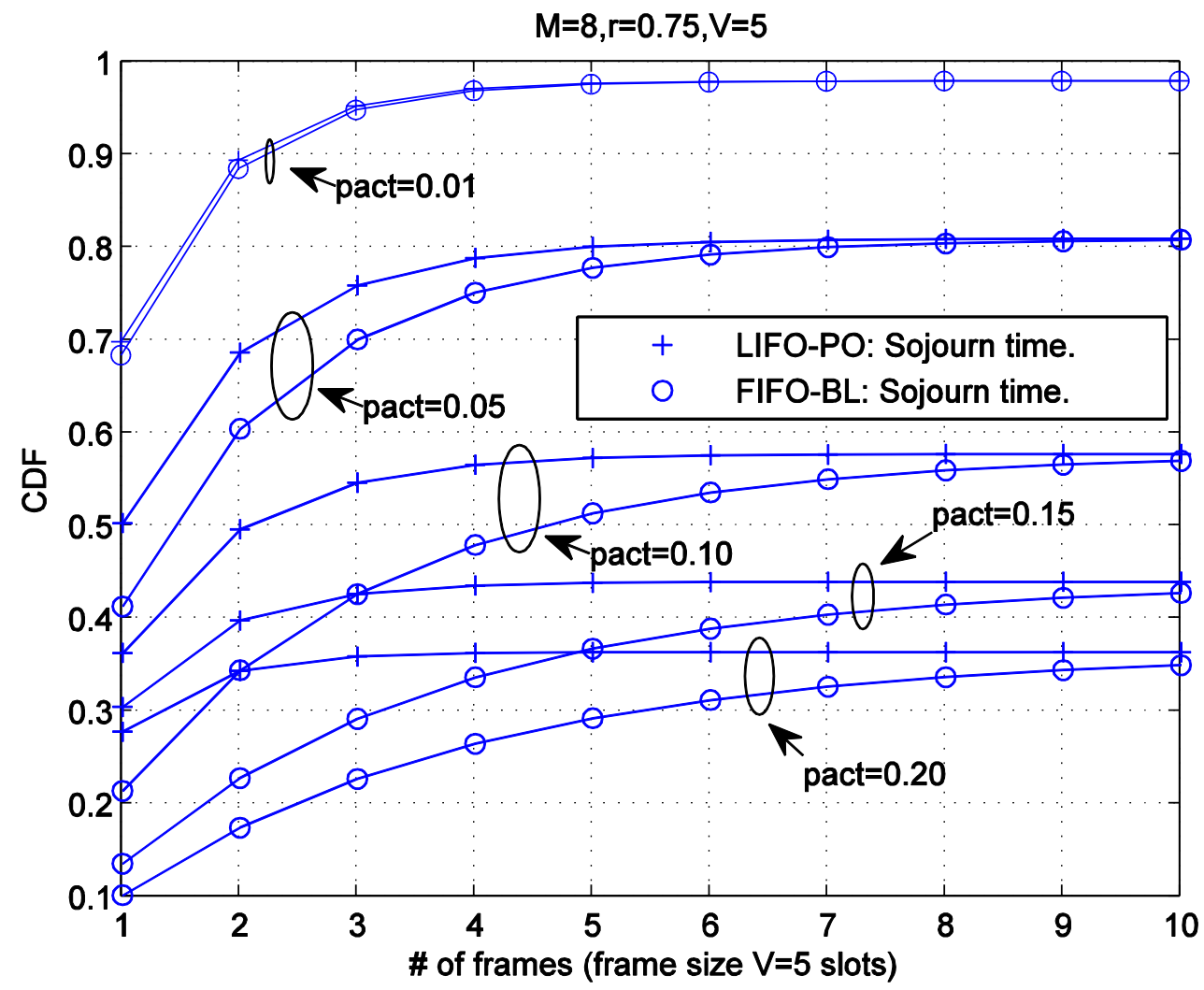

Figure 2. Cumulative Distribution Function (CDF) of the sojourn time, in number of frames, of an arbitrary packet that get a successful transmission; under FIFFO-BL and LIFO-PO disciplines for several probabilities, $P_{a c t}$, with a permission probability equal to $r=0.75$. 


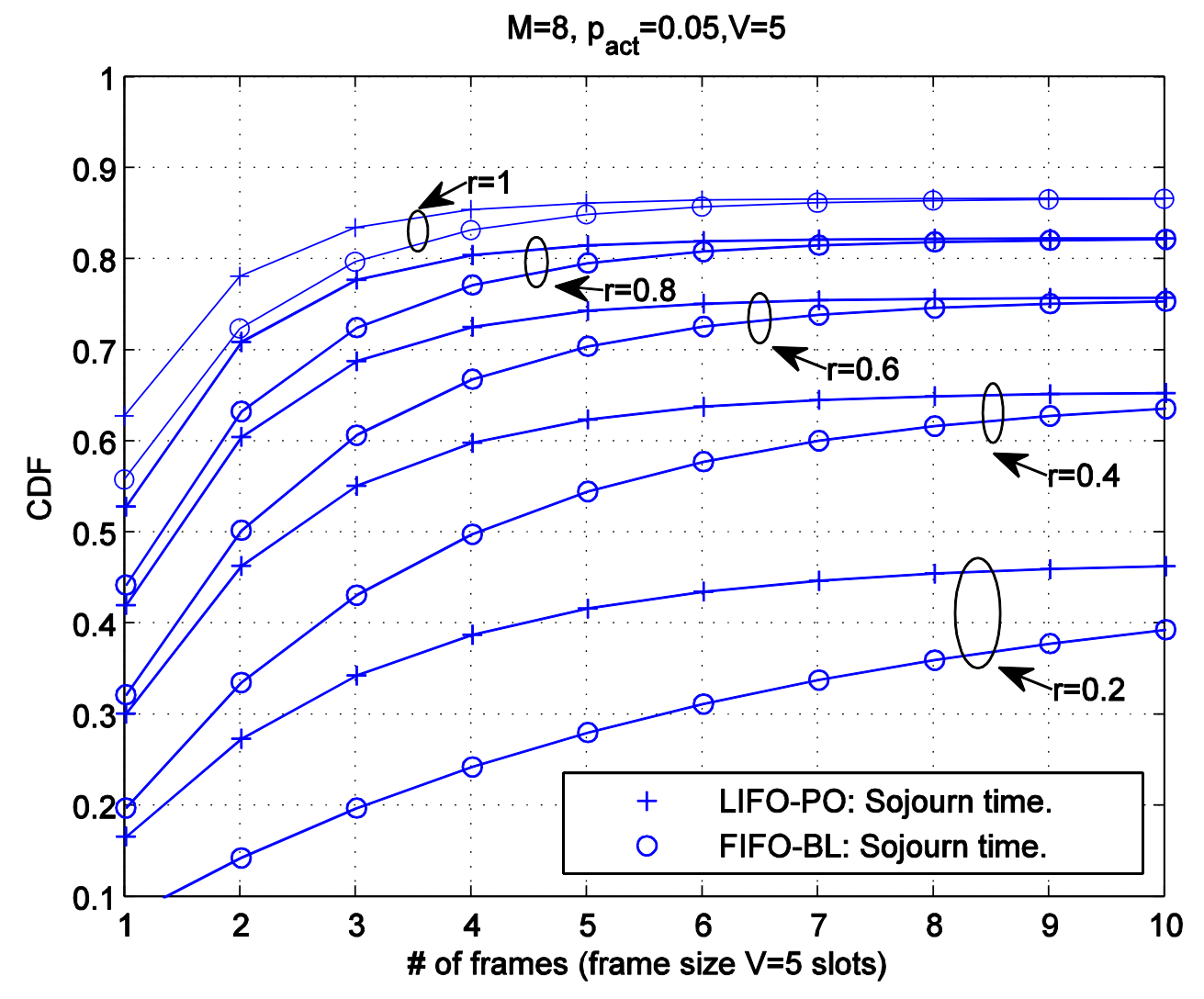

Figure 3. Cumulative Distribution Function (CDF) of the sojourn time, in number of frames, of an arbitrary packet that get a successful transmission; under FIFFO-BL and LIFO-PO disciplines for several permission probabilities, $r$, with the active probability equal to $P_{a c t}=0.05$.

First, we observe that, for a fixed value of $p_{a c t}$ in Fig. 2 or a fixed value of $r$ in Fig. 3 the asymptotical behavior of the CDFs are coincident and equal to the probability that an arbitrary tagged packet be successfully transmitted. Hence, for instance, for the mentioned traffic load $p_{a c t}=0.01,0.05,0.1,0.15$ and 0.20 , Fig. 2, the successful probability, $P_{\text {Adm }}$ in Eq (32), are $P_{A d m}=0.979,0.801,0.678,0.440$ and 0.364 , respectively; with dropped out probabilities $P_{\operatorname{Re} j}=0.021,0.199,0.422,0.560$ and 0.636 , respectively.

In Fig. 3 we observe a better behavior of the protocol for a permission probability $r=1$. This is due to the low traffic load per terminal, $p_{a c t}=0.051$, so $a=1-\left(1-p_{a c t}\right)^{V}=0.2262$, which means that, on the average, at the beginning of each frame with $V=5$ slots, we will have at least $M$ × $a=8 \times 0.2262=1.8098$ packets ready for transmission. Furthermore, we 
have to add the mean number of packets that are pending to be transmitted. Then, according to the appendix,

$$
r_{\text {opt }}=\min (1, V / m)=\min (1, V /(M \times a+\operatorname{backlog})) \leq \min (1, V /(M \times a))=\min (1,5 / 1.8098)=1
$$

Since the mean number of data packet pending to be transmitted, backlog, is bounded by backlog $<V / e-M$ х $a \approx 1.8394-1.8098=0.096$ we have $r_{o p t}=1$. Nevertheless a detailed study about the dynamic behavior of the system is needed. In other words, a parallel study to [8] should be addressed to the multi-slot scenario, where the permission probability needs to be adapted according to the observed results of the number of collision slots, successful slots and empty slots per frame of $V$ slots.

Back to the plots, we observe that the values of the CDF in the LIFO-PO case are higher than the values in the FIFO-BL case and both plots reach the asymptotic value of $P_{\text {Adm }}$. This means that the mean sojourn time of an arbitrary packet that is finally successfully transmitted is lower in the LIFO-PO case that in the FIFO-BL case. But we emphasize that we have less significant differences for low traffic load. This is due to the fact that, for such a traffic condition, in the FIFO-BL case most of the packets find a buffer empty upon its arrival and in the LIFO-PO case the push-out action is quite seldom. The difference between both disciplines increases with the traffic load but the percentage of dropping packets increases as well.

Clearly, the LIFO-PO discipline can be chosen when it is desired to transmit the most recent generated packet. Then, LIFO-PO discipline could be preferred instead of the FIFO-BL discipline where the idea of New Better that Old (NBO) or Used (NBU) [11] is a requirement in the implementation. This is the case for some applications supported by Wireless Sensor Network (WSN) where the deployed infrastructure is used to capture real time sensitive data (temperature, humidity, speed of the wind, fire detections, ...)

Finally, for the mentioned WSN scenarios we remark the applicability of the framed ALOHA protocol. In such environments, nodes or motes that are geographically close each other are clustered and between them, based on positioning and energy parameters among others, a leader, named as cluster head $(\mathrm{CH})$, is elected. With certain periodicity, the $\mathrm{CH}$ is in charge to collect the information sensed by the motes and later is being forwarded to the sink. Having in mind the low traffic load generated by each mote, protocols with low complexity are chosen for intra-cluster communications. The $\mathrm{CH}$, in addition to be one more member of the group, acts as the central manager of the communications and can provide an estimation of the permission probability $r$ to all members of the cluster. For inter-cluster communications, not treated in this work, CHs conform a mesh network and communicate between them following a multi-hop routing protocol where a TDMA like protocol becomes quite suitable or appropriated [12], [13]. 


\section{$\Lambda$ Macrothink}

\section{Conclusion}

A model to evaluate the multi slotted ALOHA protocol is formulated. Bernoulli arrival process and deterministic service time are considered. Two new results are presented. The first is the recursion formulas for the statistics about the number of slots with collisions, with successful transmissions and empties. The second result is the analysis of the FIFO-BL and LIFO-PO disciplines. For both disciplines, we have derived the Cumulative Distribution Functions (CFDs) of the sojourn time of the packets that are successfully transmitted and the packet that are dropped out. The evaluated protocol can be used in real time applications such as Wireless Sensor Network networks where random access protocols of low complexity for intra-cluster communications are required.

\section{Acknowledgement}

This work has received support from the national project TIN2013-47272-C2-1-R and from Universidad Politécnica de Valencia.

\section{References}

[1] Abramson N., "The ALOHA system-Another alternative for computer communications", in AFZPS Conf. Proc., 1970 Fall Joint Computer Conf., Vol. 37, pp. 281-285, 1970.

[2] Roberts L.G., "Extensions of packet communication technology to a hand held personal terminal", in AFZPS Conf. Proc., 1972 Spring Joint Computer Conf., Vol. 40, pp. 295-298, 1972.

[3] Rom R., Sidi M., "Multiple access protocols”, Springer-Verlag, 1989.

[4] Mouly M., Pautet M.B., “The GSM System for Mobile Communications”, Published by the authors, 1992.

[5] Szpankowski W., "Analyisis and stability considerations in a reservation multiaccess system”, IEEE Trans on Communications, Vol. 31, No. 5, pp 684-692, May 1983. http://dx.doi.org/10.1109/TCOM.1983.1095866

[6] Weiselthier J., Ephremides A., Michaels L.A., "An exact analysis and performance evaluation of framed ALOHA with capture", IEEE Trans on Communications, Vol. 37, No. 2, pp 125-137, February 1989. http://dx.doi.org/10.1109/26.20080

[7] Doshi B.T., Heffes H., "Overload performance of several processor queueing disciplines for the M/M/1 queue", IEEE Trans on Communications, Vol. 34, No. 6, pp 538-546, June 1986. http://dx.doi.org/10.1109/TCOM.1986.1096578

[8] Rivest R.L., "Network Control by Bayesian Broadcast", IEEE Trans. on Information Theory, Vol. IT-33, No. 3, pp 323-328, 1987. http://dx.doi.org/10.1109/TIT.1987.1057315 
[9] Neuts M.F., "Matrix-geometric solutions in stochastic models - An algorithmic approach". The Johns Hopkins University Press, Baltimore, 1981.

[10] Alfa A.S., "Queueing theory for telecommunications. Discrete time modelling of a single node system”, Springer, 2010.

[11] Stoyan D., "Comparison methods for queues and other stochastic models", Akademie-Verlag Berlin / John Wiley, 1983.

[12] Casares-Giner V., Felipe-Pacheco D., Todolí Ferrandis D., "Análisis y modelado en redes de sensores inalámbricas”, Actas $10^{a}$ Jornadas de Ingeniería Telemática, JITEL-2011, pp: 309-316, Universidad de Cantabria. Santander (Spain), Septiembre 28-30, 2011.

[13] Casares-Giner V., Wüechner P., Felipe-Pacheco D., de Meer H., "Combined Contention and TDMA-Based Communication in Wireless Sensor Networks", In Proc. of the 8th Euro-NF Conf. on Next Generation Internet (NGI2012), pp: 1-8, BTH, Karlskrona (Sweden), June 25-27, 2012. http://dx.doi.org/10.1109/NGI.2012.6252158

\section{Appendix}

We consider the placement of $m$ indistinguishable balls (or data packets) into $V$ indistinguishable urns (or time slots). With probability $1 / V$ each packet will choose one of the $\mathrm{V}$ slots. We can think about the following probabilistic sequential hunting procedure. With probability $1 / V$ a given tagged packet will choose the first slot and with probability $1-1 / V=(V-1) / V$ the packet will defer its choice for the next-second slot. With probability $(1-1 / V)(1 /(V-1))=1 / V$ our tagged data packet will choose the second slot and with probability $(1-1 / V)(1-1 /(V-1))=(V-2) / V$ the packet will defer its choice for the third slot. With probability $1 / V$ the packet will choose the third slot and with probability $(V-3) / V$ defer until the third slot, and so on. Obviously, this procedure guarantees that any given data packet will choose one and only one slot of the frame.

\section{A.1 Recurrences}

Let $E(m, k, V)$ denote the conditional probability that, after the random assignment, $k$ slots out of $V$ slots that conforms a frame, contain no data packet. Parallel definitions are given for the number of slots containing a single data packet, $S(m, k, V)$, and for the number of slots with more than one data packet, $C(m, k, V) . E, S$ and $C$, respectively stand from 
empty, successful and collision. Our main purpose is to obtain the set of probabilities, $E(m, k, V), S(m, k, V)$ and $C(m, k, V)$. Then, we can write

$$
E(y, k, V)=\left\{\begin{array}{l}
\sum_{j=1}^{y} B(y, j, 1 / V) E(y-j, k, V-1) \text { for } k=0 . \\
B(y, 0,1 / V) E(y, k-1, V-1)+\sum_{j=1}^{y} B(y, j, 1 / V) E(y-j, k, V-1) \text { for } k=1,2, \cdots V .
\end{array}\right.
$$

(A.1)

, where $B(M, k, a)$ is the binomial distribution,

$$
B(M, k, p)=\frac{M !}{k !(M-k) !} p^{k}(1-p)^{M-k}=C_{k}^{M} p^{k}(1-p)^{M-k}
$$

For $S(m, k, V)$ we can write

$$
S(y, k, V)=\left\{\begin{array}{l}
\sum_{j=1,0 \neq 1}^{y} B(y, j, 1 / V) S(y-j, k, V-1) \text { for } k=0 . \\
B(y, 1,1 / V) S(y-1, k-1, V-1)+\sum_{j=0, \neq 1}^{y-k} B(y, j, 1 / V) S(y-j, k, V-1) \text { for } k=1, \cdots \min (y, V) .
\end{array}\right.
$$

, and for $C(m, k, V)$ we can write

$$
C(y, k, V)=\left\{\begin{array}{l}
\sum_{j=0}^{1} B(y, j, 1 / V) C(y-j, k, V-1) \text { for } k=0 . \\
\sum_{j=0}^{1} B(y, j, 1 / V) C(y-j, k, V-1)+\sum_{j=2}^{y-k} B(y, j, 1 / V) C(y-j, k-1, V-1) \text { for } k=1,2 \cdots V .
\end{array}\right.
$$

\section{A.2 Mean values}

From the above expression we can infer the generating functions for $E(m, k, V)$ and for $S(m, k, V)$. It can be seen, after some algebra, that 


$$
\begin{gathered}
E^{*}(y, z, V)=\sum_{k=0}^{V} E(y, k, V) z^{k}=\sum_{n=0}^{V} C_{n-1}^{V-1}\left(\frac{n}{V}\right)^{y-1}(z-1)^{V-n} \\
S^{*}(y, z, V)=\sum_{k=0}^{\min (y, V)} S(y, k, V) z^{k}=\frac{V ! y !}{V^{y}} \sum_{k=0}^{\min (y, V)} \frac{(V-n)^{y-n}}{(V-y) !(y-n) !} \frac{(z-1)^{n}}{n !}
\end{gathered}
$$

Taking successive derivatives in (A.5) and (A.6) we can obtain all the factorial moments. In particular, for the mean value we have

$$
\begin{array}{r}
\frac{d E^{*}(y, z, V)}{d z}{ }_{/ z=1}=(V-1)\left(\frac{V-1}{V}\right)^{y-1} \\
{\frac{d S^{*}(y, z, V)}{d z}}_{/ z=1}=y\left(\frac{V-1}{V}\right)^{y-1}
\end{array}
$$

Then, from (A.7) and (A.8) we have

$$
\frac{d C^{*}(y, z, V)}{d z}=V-(V+y-1)\left(\frac{V-1}{V}\right)^{y-1}
$$

The throughput is maximized in (A.7) for a number of data packets $y_{\text {opt }}$ such that

$$
V-1<y_{\text {opt }}=\left(\ln \frac{V}{V-1}\right)^{-1}<V
$$

Remark: we observe that

$$
{\frac{d E^{*}(V-1, z, V)}{d z}}_{1 z=1}={\frac{d S^{*}(V-1, z, V)}{d z}}_{1 z=1}=\frac{d S^{*}(V, z, V)}{d z}{ }_{\mid z=1}
$$

For $V=3$, Fig. 4 shows the mean values of the number of slots empty, with a single data packet and with more than one data packet.

\section{A.3 Access with permission probability}

Now we consider a total of $m$ data packets that try to access in a frame of size $V$ slots. Each data packet has a permission probability equal to $r$. Then, the probability that $k$ out of $m$ get a successful access is given by 


$$
D_{k}^{m}(r, V)=\sum_{y=k}^{m} B(m, y, r) S(y, k, V) \text { for } k=0,1, \cdots, V
$$

Let $\Delta_{z}^{m}(r, V)$ denote the generating function of $D_{k}^{m}(r, V)$, that is

$$
\Delta_{z}^{m}(r, V)=\sum_{k=0}^{\min (m, V)} D_{k}^{m}(r, V) \mathrm{z}^{\mathrm{k}}
$$

After some algebra, evaluating the first derivative of (A.13) in $z=1$ we get

$$
{\frac{d \Delta_{z}^{m}(r, V)}{d z}}_{/ z=1}=r m\left(\frac{V-r}{V}\right)^{m-1}
$$

The maximum in (A.14) is achieved for $r_{o p t}$ such that

$$
r_{o p t}=\left\{\begin{array}{l}
1, \text { for } m \leq V \\
\frac{V}{m}, \text { for } V \leq m
\end{array}\right.
$$

, and the corresponding optimum throughput is given by

$$
T h_{o p t}=\left\{\begin{array}{l}
m\left(1-\frac{1}{V}\right)^{m-1}, \text { for } m \leq V \\
V\left(1-\frac{1}{m}\right)^{m-1}, \text { for } V \leq m
\end{array}\right.
$$

From (A.16) if we fix $V$ and $m \rightarrow \infty$, the permission probability goes to zero, see Eq. (A.15), and the optimum throughput goes to $V / e \approx 0.367879 \mathrm{~V}$. In other words, E-ALOHA multi-slot offers a throughput equal to the standard S-ALOHA, so no advantages are envisaged from this point of view. Nevertheless, S-ALOHA multi-slot offers another advantages and some of them has been pointed out in Section 1 . 


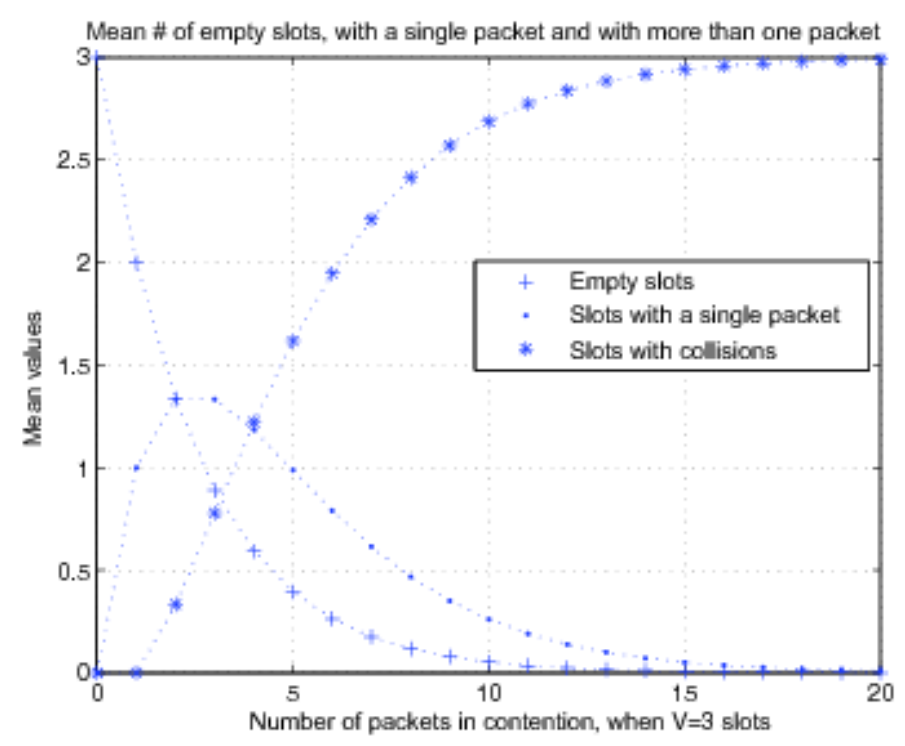

Figure 4. Mean values of the number of empty slots, successful slots and collision slots.

\section{Copyright Disclaimer}

Copyright reserved by the author(s).

This article is an open-access article distributed under the terms and conditions of the Creative Commons Attribution license (http://creativecommons.org/licenses/by/3.0/). 